\title{
Mobile Geriatric Teams - A Cost-Effective Way Of Improving Patient Safety And Reducing Traditional Healthcare Utilization Among The Frail Elderly? A Randomized Controlled Trial
}

This article was published in the following Dove Press journal:

Clinical Interventions in Aging

\section{Sofi Fristedt ${ }^{1-3}$ \\ Paul Nystedt ${ }^{4}$ \\ Örjan Skogar $\mathbb{D}^{2,5}$}

'Department of Rehabilitation and ARN-J Aging Research Network, Jönköping University, School of Health and Welfare, Jönköping, Sweden; ${ }^{2}$ Futurum - The Academy for Health and Care, Jönköping, Sweden; ${ }^{3}$ Department of Health Sciences, Lund University, Lund, Sweden; ${ }^{4}$ Jönköping Academy, Jönköping International Business School, Jönköping University, Jönköping, Sweden;

${ }^{5}$ Department of Neurobiology, Care Sciences and Society, Karolinska Institute, Huddinge, Stockholm, Sweden
Correspondence: Sofi Fristedt

School of Health and Welfare, Jönköping

University, Gjuterigatan 5, Jönköping SE

55I II, Sweden

$\mathrm{Tel}+4636-101269$

Email sofi.fristedt@ju.se
Background: Demographic changes combined with costly technological progress put a financial strain on the healthcare sector in the industrialized world. Hence, there is a constant need to develop new cost-effective treatment procedures in order to optimize the use of available resources. As a response, the concept of a Mobile Geriatric Team (MGT) has emerged not only nationally but also internationally during the last decade; however, scientific evaluation of this initiative has been very scarce. Thus, the objective of this study was to perform a mixed methods analysis, including a prospective, controlled and randomized quantitative evaluation, in combination with an interview-based qualitative assessment, to measure the effectiveness and user satisfaction of MGT.

Materials and methods: Community-dwelling, frail elderly people were randomized to an intervention group $(n=31$, mean age 84$)$ and a control group $(n=31$, mean age 86$)$. A twoyear retrospective quantitative data collection and a prospective one-year follow-up on healthcare utilization were combined with qualitative interviews. Non-parametric statistics and difference-in-difference (DiD) analyses were applied to the quantitative data. Qualitative data were analyzed using content analysis.

Results: No significant group differences in healthcare utilization were found before inclusion. Post intervention, primary care contact (including MGTs) increased for the MGT group. Inpatient care decreased dramatically for both groups. Hence, the increase in primary care contact for MGT patients was not accompanied by a reduction in inpatient care compared to the control group. Utilization of non-primary care was lower ( $\mathrm{p}<0.01)$ post-intervention in both groups.

Conclusion: There appears to be a "natural" variation in healthcare needs over time among frail elderly people. Hence, it is vital to perform open, controlled clinical studies in tandem with the implementation of new caregiving strategies. The MGT initiative was clearly appreciated but did not fully achieve the desired reduction in healthcare utilization in this study.

Trial registration: Retrospectively registered 09/10/2018, ClinicalTrials.gov ID NCT03662945.

Keywords: frail elderly, healthcare utilization, mobile geriatric team, patient safety, quality of life

\section{Background}

Demographic changes and ageing populations, in combination with costly technological progress, are putting a financial strain on the healthcare sector in the industrialized world. Hence, there is a constant need to develop new cost-effective treatment procedures in order to optimize the use of available resources. As 
illustrated in Figure 1, it is possible to meet the health needs of most older people (above the age of 65) through the ordinary healthcare system. ${ }^{1,2}$ However, there is also an increasing proportion of older people who experience an unstable state of chronic conditions, highlighted at the top of the pyramid in Figure 1. Consequently, they consume a large proportion of health and social care and are often classified as the "frail elderly". 3

There are several multidimensional factors of frailty in later life, including number of conditions, number of medications, magnitude of care, etc. ${ }^{4}$ The number of conditions is relevant insofar as multi-morbidity may have an extensive impact on health. ${ }^{4}$ In later life, strokes, myocardial infarction and chronic heart failure (CHF) are very common, ${ }^{5}$ as is dementia. ${ }^{6}$ Frailty is often combined with multiple illnesses and indirectly with polypharmacy. More than $40 \%$ of the population over 77 years of age uses more than five drugs concurrently. ${ }^{4}$ Moreover, Swedish authorities use care consumption, i.e. the extent of health and social care an individual receives, to define a term related to "frail elderly", i.e. "mest sjuka äldre" [which translates as "most sick older persons"]. " About 300,000 Swedish citizens (approximately 3\% of the total population) belong to this group. By definition, they require $\geq 25$ hours of social care, live in nursing homes for older persons, or are in need of extensive healthcare interventions. ${ }^{5}$ Finally, a recent article that included an assessment of frailty identified that the majority of older persons $(+75)$ with multimorbidity, polypharmacy and recurrent hospital admissions could be defined as frail. ${ }^{8}$ Though the exact definitions vary to some extent, there is ample empirical evidence to suggest that the frail elderly have higher mortality risks and lower remaining life expectancies than robust elderly individuals. ${ }^{9}$ Additionally, a common perception is that the health status of frail elderly persons generally deteriorates continuously (or at least does not improve significantly) for the rest of their lives, implying that healthcare costs increase. $^{10}$

In Sweden, the state is responsible for the overall healthcare policy, as supervised by the Inspectorate for Health and Care. The county councils are responsible for organizing care such that all citizens have access to good care. Municipalities are in turn responsible for the longterm health and social care of older persons, including the frail elderly. In addition, family members provide informal care. ${ }^{11}$ Furthermore, primary care and hospital-based healthcare form part of the care of older persons when needed. Primary care in Sweden is the first line of the healthcare system offering non-specialized and less advanced healthcare in primary care facilities or in the patients' homes. It is also supposed to guide the patients to the right level in the healthcare system, when necessary. Additionally, hospital admissions are often neither

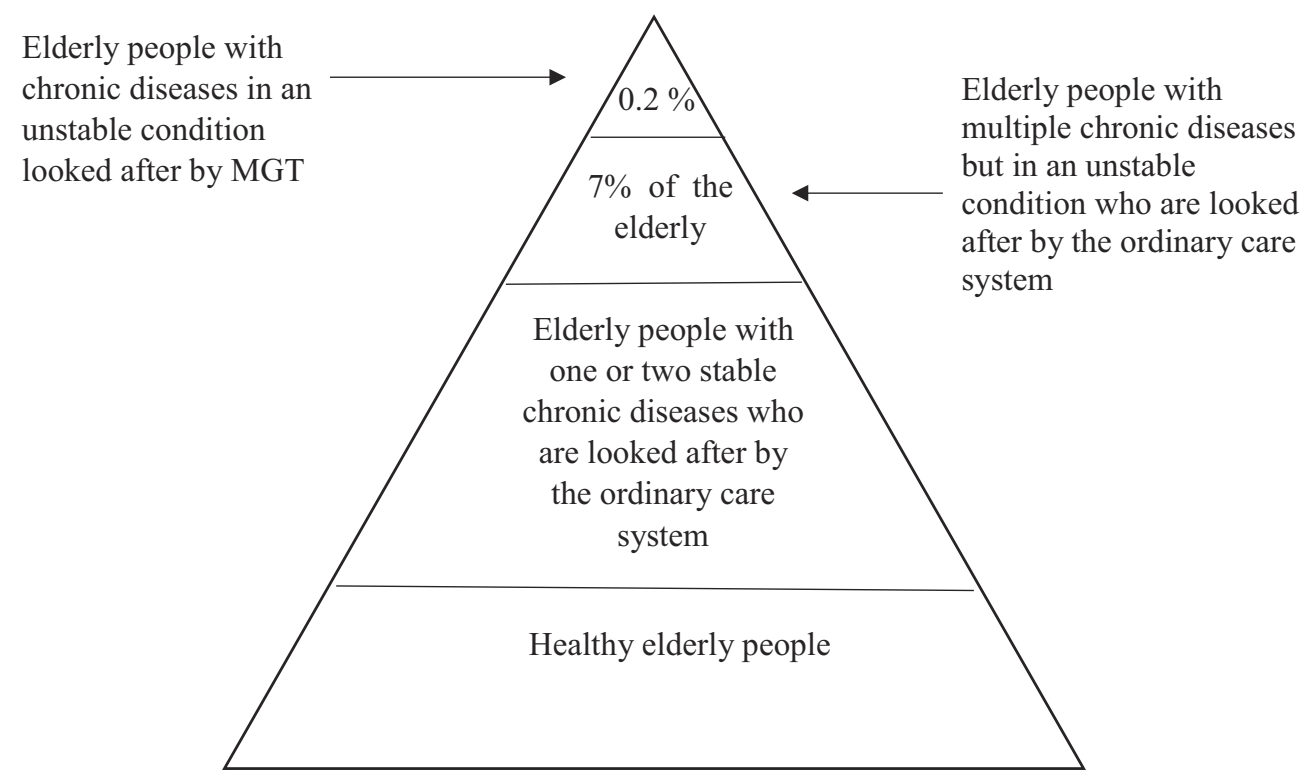

Figure I Relative proportions of degrees of chronic illnesses in an elderly population. Reproduced with permission from Lifvergren S. Quality Improvement in Healthcare. Göteborg: Chalmers University of Technology; $2013 .^{16}$ 
the best solution for nor optimized for frail elderly persons, since this target group is often misunderstood and misdiagnosed in traditional inpatient care situations. ${ }^{12}$ In addition, hospital stays are currently significantly shorter than they were some decades ago. ${ }^{13}$ Rapid changes in life situation, health status or context, in combination with a large number of healthcare providers over a short timeframe, increase the risk of mental confusion for frail elderly persons. Costly readmissions and unnecessary inpatient care can also have a negative impact on health. The relatively frequent presence of depression, low functioningand lack of social support in this group of patients are yet other important risk factors for hospital readmission. ${ }^{4}$ Thus, initial hospital admissions may cause subsequent additional and unnecessary hospitalization episodes, with further negative consequences for health among members of this group. ${ }^{12}$

In response to these challenges, a Swedish governmental initiative was launched from 2010-2014 to focus on improving the quality of life for the "most sick older persons" through coordinated care, i.e. to bridge the gap between hospital-based care and primary (including municipality-based) care. As part of this initiative, Mobile Geriatric Teams (MGT) that included physicians and nurses, as well as occupational therapists and physiotherapists at times, were created by several Swedish health and social care authorities. The aim of these teams is to conduct Comprehensive Geriatric Assessments $(\mathrm{CGA})^{14}$ in the patient's home and, based on the results of these tests, to develop as well as implement sustainable and coordinated care plans that include individually tailored interventions. These care plans are developed and implemented in collaboration with the patient, his/ her relatives and staff from the municipality. Among the primary motivations of this care concept are the improvement of communication flows between patients, their relatives and healthcare providers, in combination with the proper adaptation of the delivery of medical as well as care measures. In turn, these improvements are intended to reduce unnecessary, traditional healthcare utilizations in the form of inpatient care and EMR visits, for example. From a cost-effective perspective, MGTs should ideally be axiomatically welfare enhancing in that they improve the HRQoL of involved patients, simultaneously creating a reduction of total healthcare costs, through decreased utilization of traditional in-hospital healthcare.
While introduction of the MGT concept has emerged not only nationally but also internationally during the last decade, ${ }^{15,16}$ scientific evaluations of the initiative have been scarce. The results from a randomized controlled study of the cost-effectiveness of person-centered integrated heart failure and palliative home care did show positive welfare effects in terms of increased patient well-being and reduced hospital care utilization. ${ }^{17-19}$ However, knowledge of the economic consequences and effects on patients, relatives and other caregivers is scant.

Given the dearth of research in this regard, the objective in this study was to perform a mixed methods analysis, including a prospective, controlled and randomized quantitative evaluation, in combination with an interview based qualitative assessment, to measure the effectiveness and user satisfaction of Mobile Geriatric Teams. The term "effectiveness" is used in this manuscript to represent costeffectiveness in terms of healthcare utilization as well as user satisfaction.

\section{Materials And Methods}

The current study was conducted through a mixed methods approach including a randomized control trials (RCT) and a qualitative assessment, upon approval from the Regional Ethical Committee in Linköping, Dnr 2014/371-31, in accordance with the Declaration of Helsinki. The study context was an MGT, initiated in 2013 and originating from a hospital serving approximately 149,000 inhabitants in Southern Sweden. We do not intend to share individual deidentified participant data. Study protocol is, however, available through ClinicalTrials.gov ID NCT03662945.

\section{Participants Inclusion Criteria}

The inclusion criteria for this trial were the "frail elderly", defined as community-dwelling persons aged $>75$ years, having more than three chronic diagnoses and prescribed six or more pharmaceutical drugs for continuous use, and with $\geq$ three hospital stays ( $>24$ hours in hospital) during the last six months. Since these criteria had to be broad in order to capture relevant individuals (so as to leave no relevant persons out), but were also likely to generate persons of no relevance to MGT interventions, identification of the study sample was conducted in three steps, which were, in turn, repeated in three waves. Patients with repeated hospital admissions due to surgery (without 
multi-morbidity) or patients admitted for palliative care were also excluded from the sample.

\section{Exclusion Criteria}

The records of persons were excluded if that person was deceased, lived in a nursing home or had a hospital admission not relevant to the MGT concept (e.g. repeated hospital admissions due to surgery not indicating multi-morbidity); if an MGT would be redundant and non-relevant to offer since the patient had similar and extensive help from another caregiver; or if hospitalizations had decreased recently and the situation had been stabilized.

During the first step, a nurse with special training in identifying populations by filtered searches in digital patient records identified 449 individuals in an initial search of records (March 2015). Another 75 individuals were identified in a second search wave (May 2015), and another 157 individuals in a third search wave (September 2015) over the same collection of records.

During the second step, a nurse with geriatric competence checked the patient records of all individuals. Deceased persons or persons who had moved to a nursing home since the initial search were excluded. Patients with repeated hospital admissions due to surgery (without multi-morbidity) or patients admitted for palliative care were also excluded from the sample. Thirdly, and finally for all waves, a nurse with clinical experience in the MGT re-evaluated each of the previous searches in order to exclude individuals that an MGT would not consider for inclusion, since they were likely to not benefit from the MGT measures or for whom an MGT was irrelevant (e.g. patients with their needs satisfied by another caregiver at a hospital or in primary care, or where the hospitalizations had ceased and the situation had been stabilized). Taken together, this three-step process was considered essential to the generation of a sample similar to the ordinary target group of an MGT.

The final recruitment of participants from the filtering described above resulted in 66 potential participants from the first search, 34 from the second search and 41 from the third search (in total, 141 individuals). These individuals were consecutively numbered, from 1 to 141 on a code list. The code list was then put aside, so as to not influence the sampling. The same numbers (1-141) were written down on separate pieces of paper (one for each potential participant). The randomization was done by lottery by drawing numbers from a black box, with the first author and the research nurse present. These two individuals had no relation to the potential participants and did not have access to their names during the sampling, but merely documented the group to which each participant number was placed. To avoid putting too great of a cognitive load on potential participants, many of whom suffer from cognitive impairments, the numbers were randomized directly, as shown in Figure 2, to an intervention group and a

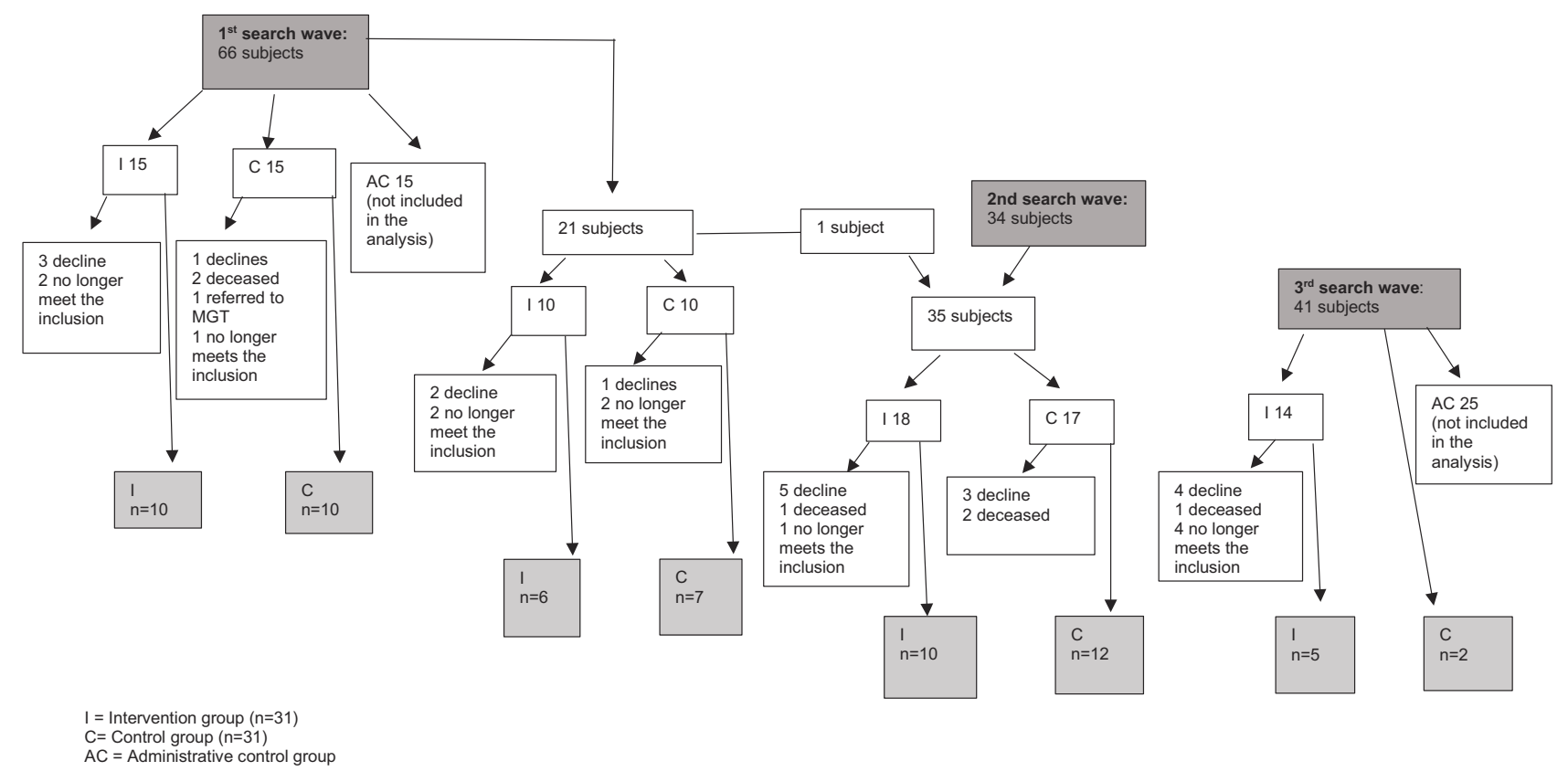

Figure 2 Flow chart on the sampling process. 
control group, comprising 31 participants each, and an administrative control group, the members of which were not contacted or followed in the present trial. The initial intention was only to observe the administrative control group data from health and social care registers, but for the purpose of the present study this was never done. Thus, the sample in the present article is based on two groups, one intervention group and one control group.

The potential participants in the control group were contacted via telephone, and the aim of the study was communicated both orally and in writing. They also gave their written, informed consent before the data collection commenced. Their families or professional caregivers were never contacted, unless their spouse was present at the interview.

Persons sampled to the intervention group were first informed on the intervention and made their decision on whether to participate or not, before being informed (orally and in writing) about the study. All persons who agreed to the MGT intervention also gave their informed consent to be part of the study (intervention group). All participants were given the chance to ask questions before their informed consent. It should be noted that this procedure departs from a traditional RCT sampling scheme insofar as (potential) participants were randomized (between an intervention and control group) before and not after enrollment. However, this was done to reduce cognitive load as described above and, thus, with ethical considerations for the participants in mind. Applying a logistic procedure like this seemed most reasonable given the context at hand. However, this process could constitute a statistical problem and bias our results if participation varied systematically between the two groups along lines that also govern the studied outcomes: how healthcare utilization develops over time. Therefore, we complemented our basic analyses with robustness checks of how any differences in individual characteristics between the two groups at baseline affected our results.

\section{Intervention}

The control group received standard care based on their individual needs from the hospital or primary care, most often also including healthcare or social care provided by the municipality or private companies. The participants in the control group had absolutely no contact with the MGT. The intervention group also received standard care from the hospital and municipality (but no primary care), as well as the MGT intervention of the present study. The
MGT-intervention was given by an experienced MGT team based at a geriatric clinic already providing the same intervention to other citizens in the same geographical area. They continued working in the same manner with the intervention group. The intervention, based on previous research, ${ }^{16}$ started with a home visit by a geriatrician and a nurse. During the first visit, a $\mathrm{CGA}^{14}$ was conducted, together with a pharmaceutical review. The kitchen or the living room were common places for the dialogue during this visit. Examination of the patient was performed in the most suitable place: in the bedroom or where conditions allowed. Based on this, a care plan that was individualized, person-centered and coordinated, as well as (hopefully) sustainable, was developed by the MGT, in collaboration with the patient, his/her relatives and the homecare staff. Since these care plans were the basis of care, specific measures in the intervention varied between clients. Overall medical responsibility for the patient was temporarily transferred to the MGT physician (the geriatrician) from primary care during the intervention period. Visits after the first one was often initiated by the patient. The initial intention was for this care to last 15 weeks, but based on the early experiences of the MGT, the time limit was removed, because it was considered inappropriate to break a continuity that added a valuable sense of security for patients. Thus, the MGT "retained" the patient as long as was considered necessary. In practice this meant that the enrollment of the MGT was not withdrawn unless the participant moved to a nursing home, stabilized, or died, and, in some individual cases, at the patient's request. If patients required palliative care, the MGT remained responsible but consulted an already-existing external palliative team, when necessary. After the initial visit, patients were free to contact the MGT during office hours (08.00-17.00) and were referred to home care or a hospital outside these hours. Additional visits from nurses, physicians or the entire team were often scheduled on a regular basis, but it was also possible to arrange these at short notice when needed, on the same day.

Fully operative and in full-time equivalents, the MGT comprised, on average, 1.95 physicians and 3.2 nurses during 2016. They cared for a stock of around 95-100 patients, matching their capacity. Based on presumed discharges, they had the capability to register an estimated 200 patients annually. The team had access to external equipment, for example, cars and mobile electronic patient records with updated lists of medication and all other information about the patient that was contained in the 
hospital patient records. The dictation of measures was performed via laptops. The main costs of the MGT initiative comprise personnel, and, in total, this amounts to around SEK 3.7 million annually (around 430,000 USD based on an exchange rate of 8.56 SEK, provided by the Swedish Central Bank for 2016), including salaries, payroll taxes and social charges. Hence, the average annual personnel cost per patient year is around SEK 37,000 SEK (or SEK 18,500 per estimated new annual registration in fully functional mode). To put this into perspective, the cost of traditional care has been calculated by the county council at, for example, SEK 6,500 for inpatient geriatric care days and SEK 1,600 for EMR (emergency room) visits. Hence, the MGT initiative must have apparent effects on traditional healthcare utilization in order to break even financially and to be self-financed.

Taking the estimated capacity of registering up to 200 patients annually literally implies that the traditional care consumption of the average MGT patient has to be reduced annually by about 2.85 care days or 11.6 EMR visits (or a combination of both) in order to reach the break-even point and be self-financed.

\section{Instruments And Variables}

Data collection was conducted retrospectively via comprehensive county council (patient records) and municipal administrative registers covering all inhabitants with regard tohealthcare consumption, home care and home help given to participants, as shown in Table 1 .

Healthcare consumption was measured via the number of EMR visits, number of hospital admissions, number of days admitted to the hospital, outpatient visits to the hospital. Primary care consumption was measured via number of visits to a) physicians and b) nurses. Note that care given by, and contact with, an MGT was not included in any of these measurements. Moreover, participants were contacted, and, during a home visit, data on performance in Activities in Daily Living (ADL) (bathing, dressing/ undressing, toileting, transfers, continence, eating) were collected through the Katz ADL index. ${ }^{20}$ In this index, ADL is measured along a seven-point scale (ie 1=independent in all activities, $2=$ dependent in one activity, $3=$ dependent in bathing and one additional activity, $4=$ dependent in bathing, dressing/undressing and one additional activity, $5=$ dependent in bathing, dressing/undressing, toileting and one additional activity, $6=$ dependent in bathing, dressing/undressing, toileting, transfers and one toileting activity, $7=$ dependent in all six activities. Moreover, cognitive function was screened using MMSE, with the scale ranging from $0-30$ points. ${ }^{21}$

Both groups were interviewed following a structured guide that covered subjective health, subjective safety and security, and their experiences of standard care and care provided by an MGT, when relevant. At inclusion, participants and relatives in the intervention group were asked to speculate on the forthcoming measures of the MGT initiative. Questions regarding experiences of the performed measures were asked and documented 15 weeks later. All face-to-face data collections were done by the project manager (first author) or (the same) research nurse (RN).

Table I Data Collection In The Intervention Group (IG) And Control Group (CG) At Different Points In Time

\begin{tabular}{|l|l|l|l|l|}
\hline & $\begin{array}{l}\text { 24/I2 Months Before } \\
\text { Inclusion }\end{array}$ & At Inclusion & $\begin{array}{l}\text { I5 Weeks After } \\
\text { Inclusion }\end{array}$ & $\begin{array}{l}\text { I2 Months After } \\
\text { Inclusion }\end{array}$ \\
\hline $\begin{array}{l}\text { Healthcare consumption from } \\
\text { patient records }\end{array}$ & $\mathrm{X} / \mathrm{X}$ & & & $\mathrm{X}$ \\
\hline MGT consumption (I) & & & & $X$ \\
\hline Home care and home help & $-/ \mathrm{X}$ & & $\mathrm{X}$ \\
\hline Katz ADL index & & $\mathrm{X}$ & \\
\hline $\begin{array}{l}\text { Mini Mental State Examination } \\
\text { (MMSE) }\end{array}$ & & $\mathrm{X}$ & $\mathrm{X}$ & \\
\hline Interview (participant \& relative) & & $\mathrm{X}$ & $\mathrm{X}$ & \\
\hline
\end{tabular}

Notes: * no. EMR visits, no. hospital admissions, no. days admitted to hospital, no. outpatient visits hospital, no. primary care visits. 


\section{Analysis}

Variation in healthcare utilization pre and post MGT intervention was compared for the control and the intervention group, respectively, as well as between groups pre- and post-intervention via two-sample Wilcoxon rank-sum (Mann-Whitney) tests. In order to discern whether the MGT treatment had any causal effects on healthcare utilization, the data were assessed via a difference-in-difference (DiD) analysis, in which the change in utilization following the introduction of the MGT in the treatment group was compared to the corresponding development of the unaffected control group during the same timeframe. In short, the DiD implies that the difference pre- and postintervention in the outcome under study for an "untreated" control group is subtracted from the corresponding difference for the treated group. The result yields a so-called "difference-in-difference" estimate of the treatment. It should be noted that the underlying basic assumption of the DiD is that the change in outcome for the treated group, had they not been treated, would have mirrored the change that occurs in the control group, not that the two groups are necessarily equal from every respect at baseline. The actual DiD estimate could be obtained by the following simple regression: $\Delta y_{i}=\beta_{0}+\beta_{1} D_{i}+e_{i}$

Where $\Delta y_{i}$ is the actual difference in outcomes between baseline and follow-up for individual $i, \beta_{0}$ represents a time trend that would affect the outcome regardless of an individual being treated or not, $e_{i}$ is the error term, $D_{i}$ is an indicator variable for treatment, and the hereto related parameter $\beta_{1}$ represents the expected additional change in outcome for the treated, or, in other words: the differencein-difference between the treated and untreated.

The DiD analysis is estimated via linear regression, and the reported significance values are obtained using robust standard errors.

The qualitative interviews were transcribed verbatim. The analysis was done inductively, focusing on the manifest content. ${ }^{22}$ The qualitative analysis was mainly done by the first author but was discussed with the two coauthors. The transcripts were read in full-text several times, to get a sense of the material. Meaning units focusing on the participants' experience of care (standard care, as well as MGT) were identified in the transcripts. In general, the participants had difficulty rating or describing their health or sense of security; thus, meaning units relating to these issues were not included in the present study. As a next step, the chosen meaning units were condensed, i.e. shortened, with the intention of retaining the original meaning. These condensed meaning units were assigned content-describing codes. These codes were read, compared as well contrasted and finally abstracted into two categories. These categories were formed based on similarities as well as differences and given content-describing names. $^{22}$

\section{Results}

Demographic descriptive statistics for the intervention group and the control group are presented in Table 2 . Overall, there were no statistically significant distributional differences between the characteristics (age, gender, housing, living area, education, number of prescribed drugs, MMSE and Katz) of the intervention group and the control group, with two exceptions. Firstly, the intervention group had an average of 1.3 more conditions than the control group (6.75 vs. 5.45). All participants had cardiovascular conditions. Orthopedic conditions and respiratory conditions were also quite common. Secondly, participants in the intervention group were cohabiting to a larger extent than participants in the control group. Finally, there was no significant variation in mortality between the two groups during the one-year followup, as eight individuals in the intervention group and six individuals in the control group died during this period (see last row of Table 2).

Having presented these descriptive statistics, the qualitative findings will now be described, followed by the quantitative analysis relating to the group and over time.

\section{Qualitative Findings}

Two main categories were identified: "Hospital-based and home care is appreciated, but primary care and home helps leaves a lot to be desired" and "MGT - an intervention valued beyond prior expectations". These are described below.

Hospital-based and home care is appreciated, but primary care and home helps leaves a lot to be desired

Overall, the participants in both groups were satisfied with the hospital care they had received, both at inclusion and 15 weeks after inclusion. Their views on ordinary primary care were less positive. The high turnover rate of physicians, implying poor continuity, was commonly perceived as problematic. Moreover, several participants found it disadvantageous that they could not address more than one problem at a time with their physician. Raising additional (often related) problems during a primary care 
Table 2 Background Variables In The Intervention Group (IG) And Control Group (CG), And Group Comparisons Between The IG And CG

\begin{tabular}{|c|c|c|c|}
\hline & $\begin{array}{l}\text { IG } \\
(n=3 I)\end{array}$ & $\begin{array}{l}\text { CG } \\
(n=31)\end{array}$ & $\begin{array}{l}\text { Group } \\
\text { Comparisons } \\
\text { z-value/p-value }\end{array}$ \\
\hline Age Mean (SD) & $84(5.1)$ & $86(5.7)$ & $-1.17 / 0.24$ \\
\hline Gender M/F & $16 / 15$ & $12 / 19$ & $-\mid .01 / 0.31$ \\
\hline $\begin{array}{l}\text { Marital status } \\
\text { Married } \\
\text { Never married } \\
\text { Widowed }\end{array}$ & $\begin{array}{l}11 \\
5 \\
7\end{array}$ & $\begin{array}{l}12 \\
3 \\
16\end{array}$ & $-1.69 / 0.09$ \\
\hline Co-habiting Yes/No & $16 / 11$ & $11 / 19$ & $-2.10 / 0.04$ \\
\hline $\begin{array}{l}\text { Housing } \\
\text { House } \\
\text { Apartment }\end{array}$ & $\begin{array}{l}5 \\
25\end{array}$ & $\begin{array}{l}5 \\
25\end{array}$ & $0.00 / 1.00$ \\
\hline $\begin{array}{l}\text { Living area } \\
\text { Urban } \\
\text { Suburban } \\
\text { Rural }\end{array}$ & $\begin{array}{l}21 \\
8 \\
2\end{array}$ & $\begin{array}{l}18 \\
11 \\
1\end{array}$ & $-0.50 / 0.62$ \\
\hline $\begin{array}{l}\text { Education } \\
\text { Primary school } \\
\text { Secondary school } \\
\text { High school } \\
\text { University }\end{array}$ & $\begin{array}{l}17 \\
5 \\
6 \\
1\end{array}$ & $\begin{array}{l}21 \\
4 \\
4\end{array}$ & $-1.18 / 0.24$ \\
\hline $\begin{array}{l}\text { No. diagnoses Mean } \\
\text { (SD) }\end{array}$ & $6.84(2.3)$ & $5.40(1.7)$ & $-2.64 / 0.08$ \\
\hline $\begin{array}{l}\text { No. of drugs Mean } \\
(\min / \max )\end{array}$ & $10.4(4 / 17)$ & $10.3(6 / 14)$ & \\
\hline $\begin{array}{l}\text { MMSE Median } \\
\text { (IQR) }\end{array}$ & $25.61(3.3)$ & $26.87(2.86)$ & $-1.66 / 0.10$ \\
\hline Katz Median (IQR) & $2.10(1.42)$ & $2(1.21)$ & $-0.02 / 0.98$ \\
\hline $\begin{array}{l}\text { No. of deceased } \\
\text { during follow-up }\end{array}$ & 8 & 6 & $0.00 / 1.00$ \\
\hline
\end{tabular}

visit was usually not allow, and the participants had to make another appointment to see about these additional problems. Language issues relating to consultations with foreign-born physicians were also considered problematic by some participants. Participants who experienced higher degrees of continuity over time were more satisfied than the others.

While the home care nurses were highly valued, the participants often raised issues relate to home help. Some participants considered it problematic that home help staff did not have sufficient time (or knowledge) to conduct household chores "properly", while others accepted the "staff" way of doing things, and still others were very satisfied with the service they received. It was also considered problematic that the home help moved things, or that older persons had to show them what to do and how to do it, and that they did not arrive punctually. Some participants appeared to think that the home help's performance had individual variance, exemplified by one participant:

It [home help service] depends on who's coming in terms of behavior, conduct, knowledge and ineptitude. There were no obvious differences of opinion between the intervention group and the control group in this regard.

\section{MGT - An Intervention Valued Beyond Prior Expectations}

The MGT intervention was appreciated for providing services at home. Frail elderly persons found it energydepleting to leave their homes for a healthcare visit, and some actually stated they had no energy at all to visit a physician. Having access to an MGT meant not having to prepare themselves to go out (to shower, etc.), nor having to depend on someone to accompany them or drive them. One participant stated the following:

Older persons are more concentrated [when going to the doctor] and you don't have to be that at home. The MGT way of working from the kitchen table levelled the balance of power in the situation; Mark and Mary [fictional names of MGT members] have a humble attitude and they don't sit behind a desk. At this table [kitchen table] the situation becomes totally different.

Supporting this thought another person said:

When the doctor visits me at home it becomes easier, and a different situation. Then you are someone. It's me they are going to do something with. You don't get that feeling when visiting an institution.

Moreover, the MGT provided continuity as well as safety and security, by working as a small team, visiting or calling as promised, and behaving in a serviceminded manner. Overall, the intervention group seemed to think that the MGT staff listened to them properly and clearly involved them in care decisions. More often than other caregivers (that the participants had recently experienced), the MGT did not make a conclusion about an issue until it had been sufficiently studied, and the 
team also kept their promises. Due to the risk of having to wait at the EMR and meeting non-familiar staff, the frail elderly persons (in general) often hesitated before calling, hoping that the problem would simply go away. The participants in the intervention group instead appeared to call the team earlier, with less severe symptoms, than if their only option was the EMR. Thus, interventions could be provided earlier and hospitalization avoided, the participants concluded. One clear benefit, which was not expressed by the participants, but deserves to be mentioned here, was that the service from the MGT was free of charge. One disadvantage that became apparent was the fact that the MGT operated only during office hours, not evenings, nights or weekends. Some participants also wanted the MGT to visit more often than once a month, for example.

\section{Quantitative Findings}

The main results of the quantitative study are presented in Table 3. The first two panels present the average annual healthcare utilization for the two groups one year before and after the intervention and note whether there are any discrepancies between them in this respect. Note that the figures of primary care utilization do not include any MGT-related healthcare contacts, and that, by definition, there are no such contacts before the intervention.

Overall, the utilization before intervention was rather similar between the two groups, and there are no significant differences in this respect (first panel), though it should be noted that the average number of outpatient hospital care episodes in the control group exceeded the MGT group by 1.48. During the intervention one-year follow-up period, healthcare utilization was also similar

Table 3 Mean Values And Difference-In-Difference Estimates Of Healthcare Utilization Among Intervention Group (IG) And Control Group (CG) Patients Before And After The MGT Intervention

\begin{tabular}{|c|c|c|c|c|c|c|}
\hline & \multirow[t]{2}{*}{ EMR } & \multirow[t]{2}{*}{ Hospital Outpatient } & \multirow[t]{2}{*}{ Hospital Admissions } & \multirow[t]{2}{*}{ Care Days } & \multicolumn{2}{|c|}{ Non-MGT Primary Care } \\
\hline & & & & & Physician & Nurse \\
\hline \multicolumn{7}{|l|}{ Before } \\
\hline IG & 5.065 & 4.806 & 5.194 & 33.387 & 5.129 & 3.677 \\
\hline CG & $5.58 \mathrm{I}$ & 6.290 & 5.226 & 34.419 & 4.613 & 2.677 \\
\hline Diff (MGT-C) & -0.516 & -1.484 & -0.032 & -1.032 & 0.516 & 1.000 \\
\hline$P$-value & 0.891 & 0.515 & 0.875 & 0.927 & 0.283 & 0.774 \\
\hline \multicolumn{7}{|l|}{ After } \\
\hline IG & 1.742 & 1.742 & 1.903 & 14.968 & 0.710 & 0.645 \\
\hline CG & 2.323 & 3.129 & 1.968 & 14.710 & 3.226 & 1.419 \\
\hline Diff (MGT-C) & -0.581 & -1.387 & -0.065 & 0.258 & -2.516 & -0.774 \\
\hline P-value & 0.607 & 0.475 & 0.578 & 0.352 & $<0.001$ & 0.015 \\
\hline \multicolumn{7}{|c|}{ Difference: After-Before } \\
\hline IG & -3.323 & -3.064 & -3.291 & -18.419 & -4.419 & -3.032 \\
\hline P-value & $<0.0001$ & 0.0001 & $<0.0001$ & $<0.0001$ & 0.0000 & 0.0001 \\
\hline Control & -3.258 & -3.161 & -3.258 & -19.709 & -1.387 & -1.258 \\
\hline P-value & $<0.0001$ & 0.0014 & $<0.0001$ & 0.0001 & 0.039 & 0.599 \\
\hline \multicolumn{7}{|l|}{ Diff-in-diff } \\
\hline$(\Delta M G T-\Delta C)$ & -0.065 & 0.097 & -0.032 & 1.290 & -3.032 & -1.774 \\
\hline Std. error & 1.262 & 1.528 & 0.746 & 7.110 & 0.896 & 1.212 \\
\hline P-value & 0.959 & 0.950 & 0.966 & 0.856 & 0.001 & 0.146 \\
\hline Diff-in-diff* & \multicolumn{6}{|c|}{ Controlling for number of chronic condition and cohabitation at baseline } \\
\hline$(\Delta \mathrm{MGT}-\Delta \mathrm{C})$ & 0.209 & 0.946 & 0.162 & 5.364 & -3.379 & $-1.14 \mid$ \\
\hline Std. error & 0.859 & 1.033 & 0.905 & 8.403 & 0.803 & 0.930 \\
\hline P-value & 0.809 & 0.365 & 0.859 & 0.526 & $<0.001$ & 0.225 \\
\hline
\end{tabular}

Notes: Figures based on 30 individuals in the CG and 32 individuals in the IG. Reported p-values for the difference between the CG and IG before and after the MGT intervention, and the difference before and after the intervention for the two respective groups are based on Wilcoxon-Mann-Whitney rank-sum (WMW) tests. Standard Errors and $\mathrm{p}$-values for the Difference-in-Difference analysis are estimated by linear regression with robust standard errors. *Note that the results from the DiD estimates controlling for number of chronic condition and cohabitation at baseline are only presented in terms of the resulting DiD ( $\Delta M G T-\Delta C)$ parameter estimate. 
between the two groups with regard to non-primary or hospital-based care: EMR visits, outpatient visits to the hospital, and number of hospital admissions and care days (days admitted to hospital). Focusing on the difference between the two periods within each group (panel 3), the utilization of non-primary care is consistently and significantly lower post-intervention in both groups. The average number of EMR visits, outpatient visits to the hospital, number of hospital admissions and care days decreased by about three EMR visits for each group, and the number of days admitted to hospital dropped by 18.4 days in the intervention group and 19.7 days in the control group. Taken together, non-primary healthcare episodes more than halved between the two periods for both groups. All these changes are statistically significant $(p<0.01)$.

Primary healthcare utilization (physicians, nurses) was slightly (but non-significantly) higher in the intervention group pre-intervention (5.12 vs. 4.61 and 3.68 vs. 2.68 for physician and nurse visits, respectively). The intervention group experienced an average of 11.16 physician and 4.04 nurse care contacts with MGT staff (result not shown), and the non-MGT primary care contacts were consequently limited to 0.71 and 0.64 , respectively, for this group. The primary care contacts fell to 3.22 (physicians) and 1.42 (nurses) in the control group. Overall, the MGT intervention meant that the total number of primary care contacts with physicians (MGT + Ordinary) increased from 5.12 to 11.87 in the intervention group and fell from 4.61 to 3.22 in the control group. The corresponding figures for contacts with nurses was 3.68 to 4.68 and 2.68 to 1.42 , respectively. Hence, the MGT intervention overall raised the frequency of contact between patients and primary care agents significantly, especially with physicians, while decreasing the utilization of ordinary primary care.

Now, turning to the core of our study, the analysis of the difference-in-difference between the two groups, it becomes evident that the changes in healthcare utilization are very similar between the two groups with regard to the non-primary care measures: EMR visits, outpatient care, number of hospital admissions and care days (see panel 4). While the average number of the first three of these decreased similarly in both groups (the difference-in-difference is less than 0.1 occasions in absolute terms), the number of care days decreased by about 1.3 instances more in the control group, although none of these differences in changes are not statistically significant in the least. The ordinary primary care visits decreased more in the intervention group (statistically significant for physician visits, but insignificant for nurse visits) as a consequence of the MGT initiative replacing it. In total, primary care contacts (MGT and traditional primary care) increased more in the MGT group. However - and contrary to the intentions of the intervention - this increase did not mean that the utilization of hospital-based care decreased more in the intervention group compared to the control group. As noted in the descriptive statistics above, the MGT and intervention group differed at baseline in terms of frequency for chronic conditions and cohabitation. Although these do not seem to have affected the studied healthcare utilization at baseline markedly, they could still potentially be related to the change in healthcare utilization, biasing our results. To address whether this is the case, we re-estimated all DiD estimates controlling for these two characteristics. The resulting point estimates indicate that all non-primary healthcare utilization rates decreased faster in the control than in the MGT group, though none of these estimates are statistically significant (see panel 5). Hence, the results of healthcare utilization rates not decreasing faster in the intervention group than in the control group are robust against initial variations in chronic conditions and cohabitation between the two groups.

Variations in mortality risks between the two groups may dilute (or confound) the results if, for instance, the survival probabilities of MGT patients are elevated, implying that, for example, the total days of exposure (i.e. length of life) is higher for them. Another potential mortality riskrelated confounder would arise if healthcare costs increase drastically close to death and the MGT intervention affects survival one way or the other. As previously shown, the mortality risk during the year of intervention is fairly similar in the two groups (see last row, Table 2). However, due to mortality not being identically distributed within the two groups, the accumulated days at risk during the pre- and post-intervention periods differ. The fact that healthcare utilization is lower during the second period could also partly be due to people dying. It should be noted that discarding observations due to mortality within the observed timeframe implies conditioning on the future and may yield biased estimates. Nevertheless, for illustrative reasons, the results for the subsample conditioning on survival during the intervention year, corresponding to Table 3 above, are shown in Table 4. Six people in the control group and eight people in the intervention group died during the one-year follow-up, leaving 25 and 23 surviving individuals in each group, respectively. The 
Table 4 Mean Values And Difference-In-Difference Estimates Of Healthcare Utilization Among Intervention Group (IG) And Control Group (CG) Before And After The MGT Intervention. Sample Restricted Upon Survival One-Year Post Intervention

\begin{tabular}{|c|c|c|c|c|c|c|}
\hline & \multirow[t]{2}{*}{ EMR } & \multirow[t]{2}{*}{ Hospital Outpatient } & \multirow[t]{2}{*}{ Hospital Admissions } & \multirow[t]{2}{*}{ Care Days } & \multicolumn{2}{|c|}{ Non MGT Primary Care } \\
\hline & & & & & Physician & Nurse \\
\hline \multicolumn{7}{|l|}{ Before } \\
\hline IG & 5.087 & 4.609 & 5.348 & 33.913 & 5.000 & 3.783 \\
\hline CG & 5.800 & 6.760 & 5.280 & 34.200 & 3.960 & 2.640 \\
\hline Diff (MGT-C) & -0.713 & -2.151 & 0.068 & -0.287 & 1.040 & 1.143 \\
\hline $\mathrm{P}$-value & 0.825 & 0.285 & 0.690 & 0.820 & 0.155 & 0.489 \\
\hline \multicolumn{7}{|l|}{ After } \\
\hline IG & 1.913 & 2.000 & 2.000 & 14.000 & 0.739 & 0.739 \\
\hline CG & 2.400 & 3.720 & 2.080 & 13.280 & 3.320 & 1.640 \\
\hline Diff (MGT-C) & -0.487 & -1.720 & -0.080 & 0.720 & -2.581 & -0.901 \\
\hline$P$-value & 0.468 & 0.450 & $0.74 I$ & 0.618 & $<0.000$ I & 0.015 \\
\hline \multicolumn{7}{|l|}{ Difference: After-Before } \\
\hline IG & -3.174 & -2.609 & -3.348 & -19.913 & -4.261 & -3.04 \\
\hline P-value & $<0.0001$ & 0.0043 & $<0.0001$ & 0.0006 & $<0.0001$ & 0.0002 \\
\hline Control & -3.4 & -3.04 & -3.2 & -20.92 & -0.64 & -1.00 \\
\hline P-value & 0.0002 & 0.0067 & $<0.0001$ & 0.0003 & 0.1354 & 0.3277 \\
\hline \multicolumn{7}{|l|}{ Diff-in-diff. } \\
\hline$(\Delta \mathrm{MGT}-\Delta \mathrm{C})$ & 0.226 & 0.431 & -0.148 & 1.007 & -3.621 & -2.043 \\
\hline Std. error & 1.543 & 1.810 & 0.896 & 7.959 & 0.962 & 1.475 \\
\hline P-value & 0.884 & 0.812 & 0.869 & 0.900 & $<0.001$ & 0.169 \\
\hline Diff-in-diff & \multicolumn{6}{|c|}{ Controlling for number of chronic condition and cohabitation at baseline } \\
\hline$(\Delta M G T-\Delta C)$ & 0.629 & 1.031 & 0.194 & 8.659 & -3.810 & -1.846 \\
\hline Std. error & 0.942 & 1.104 & 1.119 & 9.077 & 0.782 & 1.069 \\
\hline P-value & 0.508 & 0.357 & 0.863 & 0.345 & $<0.001$ & 0.091 \\
\hline
\end{tabular}

Notes: Figures based on 24 individuals in the control group and 24 individuals in the IG who survived I year after MGT start. Reported p-values for the difference between the CG and IG before and after the MGT intervention, and the difference before and after the intervention for the two groups are based on Wilcoxon-Mann-Whitney ranksum (WMW) tests. Standard Errors and p-values for the Difference-in-Difference analysis are estimated by linear regression with robust standard errors.

resulting parameter estimates (Table 4) hardly change at all, which suggests that variation in mortality rates does not affect the results.

The fact that healthcare utilization in this vulnerable group of the frail elderly has decreased over time overall is striking and may seem somewhat counterintuitive. In order to more closely analyze the hospital-based healthcare utilization pattern over time, we extended the study period backwards in time, adding information for the studied individuals from the previous year (i.e. the year before our original "one year before intervention" period). The results are shown in Tables 5 and 6 for the full sample and for the individuals surviving the one-year follow-up, respectively.

Overall, annual average healthcare utilization is similar two years before the intervention and during the one-year follow-up. Hence, the anomaly, in this respect, is the high utilization during the year preceding the intervention. This implies that there is a rather strong "natural" variation in the need for healthcare (and implicitly also, in health status) among the frail elderly who are still living at home.

\section{Discussion}

The purpose of this study was to evaluate the cost-effectiveness of MGTs by performing a prospective and randomized controlled trial. Mixing qualitative and quantitative methods added valuable information and made it possible to respond to the purpose from different perspectives and on different levels, as previously suggested. ${ }^{23}$

The qualitative findings show that this intervention was appreciated and perceived as welfare enhancing by the patients and their relatives, similar to previous research. ${ }^{18,19}$ The continuity, energy preservation and the levelling of the balance of power through consultations at the kitchen table were appreciated, and appeared to contribute to valuable 
Table 5 Annual Healthcare Utilization (Mean Values) I And 2 Years Before, And I Year After Study Inclusion In Intervention Group (IG) And Control Group (CG)

\begin{tabular}{|l|l|l|l|l|}
\hline \multirow{2}{*}{} & & \multicolumn{3}{|c|}{ Years To/From Intervention } \\
\cline { 3 - 5 } & & -2 & $-I$ & I \\
\hline EMR & IG & 2.13 & 5.06 & 1.74 \\
& CG & 2.97 & 5.58 & 2.32 \\
\hline Hospital outpatient & IG & 5.26 & $4.8 I$ & 1.74 \\
& CG & 3.23 & 6.29 & 3.13 \\
\hline Hospital admissions & IG & 1.81 & 5.19 & 1.90 \\
& CG & 1.52 & 5.23 & 1.97 \\
\hline Care days & IG & 10.58 & 33.39 & 14.97 \\
& CG & 7.68 & 34.42 & 14.71 \\
\hline
\end{tabular}

Table 6 Annual Healthcare Utilization (Mean Values) I And 2 Years Before, And I Year After Study Inclusion. Sample Restricted Upon Survival One-Year Post Intervention

\begin{tabular}{|l|l|l|l|l|}
\hline \multirow{2}{*}{} & & \multicolumn{3}{|c|}{ Years To/From Intervention } \\
\cline { 3 - 5 } & & -2 & $-I$ & I \\
\hline EMR & IG & 2.09 & 5.09 & 1.91 \\
& CG & 3.52 & 5.80 & 2.40 \\
\hline Hospital outpatient & IG & 5.61 & 4.61 & 2.00 \\
& CG & 2.76 & 6.76 & 3.72 \\
\hline Hospital admissions & IG & 1.83 & 5.35 & 2.00 \\
& CG & 1.72 & 5.28 & 2.08 \\
\hline Care days & IG & 9.74 & 33.91 & 14.00 \\
& CG & 8.96 & 34.20 & 13.28 \\
\hline
\end{tabular}

empowerment. ${ }^{24}$ Our initial intention was to let the participants rate their own health, safety and security, quantitively. However, probably because of their condition (i.e. being frail elderly persons), it transpired that it was too difficult for the participants to perform this rating without a great deal of prompting from the interviewers. Thus, we refrained from analyzing this kind of information and focused instead on qualitative descriptions. Similar challenges were described previously. ${ }^{25}$ Nevertheless, research targeting interventions for the frail elderly is of vital importance to improving healthcare. $^{25}$

Quantitatively, the MGT intervention increased the patients' primary care contacts overall, in line with its intentions. However, it did not have the intended effects during the follow-up as far as hospital-based healthcare utilization was concerned. Previous research in this area has achieved mixed results, ${ }^{18,19,26}$ which could be due to the different characteristics and contexts of the interventions under study. For instance, Ekdahl et al (2015), studying an ambulatory geriatric unit, found that the number of hospitalizations did not differ, but that the intervention group had fewer care days within these hospitalizations. In our sample, the intervention group and the control group had similar hospital utilization rates, pre- as well as post-intervention, and there were absolutely no discernable difference-in-difference results between the two groups. One reason for this may obviously be that the MGT intervention had no effect on the health and care needs of the patients. But it could also be that the closer relationships between patients and healthcare providers, induced by the facilities of the MGT, mean there is more careful health monitoring. This could, in turn, increase the probability of being referred to a physician as a consequence of different health indicators being triggered. If this is the case, positive effects on health induced by an intervention are not necessarily followed by a proportional reduction in healthcare utilization. The resulting consequences for healthcare utilization, or rather a lack thereof, though unintentional, are hence not necessarily a bad thing, but could rather constitute a sign of healthcare quality.

However, from our perspective, and most importantly, the implications of the findings of this study extend beyond the specific case into a more general arena. Firstly, this study extends the knowledge on sustainable research strategies using the frail elderly as participants, as previously called for, ${ }^{25}$ as it shows that it is indeed possible to perform clinical science in populations comprising the frail elderly, a group that were difficult to identify in the era before the digital registration of multiple characteristics of complex needs and behaviors. Our initial presumption was that controls would be very difficult, if not impossible, to recruit from among the frail elderly, but this proved to be wrong. The recruitment process was complicated due to the complexity of the included variables potentially interfering with the chosen outcome measures. Nevertheless, this study shows that a well-planned process that includes the randomization of voluntary participants works well with regard to analyzing the impact of interventions among frail elderly people.

Secondly, it is often presumed that frail elderly people with multiple diagnoses and frequent healthcare utilizations will not recover, implying that any health improvements following intervention in this group could be perceived as causal. On the contrary, we find that the 
"most sick older persons", as identified by number of diagnoses and healthcare utilization rates during a year, who actually survive during this year are also, on average, somewhat less frail (by the same definition) the following year (during follow-up). If the "frailty level" as defined by administrative health records varies to at least some extent randomly over time (though the overall trend may be negative), evaluation studies sampling from any end of the distribution (e.g. the frailest elderly) risk being plagued by "regression towards the mean". In this case, the studied individuals could be expected to "improve" along (all or some of) the considered frailty "dimensions", consuming less care, regardless of whether they were subjected to an intervention or not. The difference-in-difference procedure employed here effectively neutralizes the influence of such trends. Overall, this highlights the importance of not taking positive changes as indicators of intervention effects, thereby emphasizing the need for controlled studies in this particular group, as well.

Taking the means into consideration (Table 2), drug use was similar in both groups. However, the MGT intervention implied that medication was reflected upon and revised, resulting in changes rather than a reduction in medication. Hopefully, assessments and changes resulted in a better fit of drugs for each patient.

For practical reasons a blinded outcome assessment was not possible to include, and this is a limitation. However, using mixed methods is relevant when evaluating complex interventions, ${ }^{27}$ such as an MGT intervention for the "most sick older persons". Although participants were able to participate in our interviews, responses were sometimes quite short and underdeveloped, perhaps because respondents were frail, older persons. Nevertheless, we think the information adds to the manuscript, especially since it represents user perspectives. All text describing the qualitative findings are based on statements made by the participants.

Although we found no evidence of any intended effects on hospital-based healthcare utilization during the oneyear follow-up that was studied, the intervention may have positive (or negative) effects over the longer term. Thus, future studies in the field could benefit from high volume and a longer observation time. On a speculative basis, an increase in at subjectively experienced sense of security and safety shown in the qualitative analyses, in combination with an increased frequency in total primary care patient-physician contact (including MGT), could affect health and mortality in the longer term.

\section{Conclusions}

This study confirms that it is not only possible but also of vital interest to perform open, controlled clinical studies when new, previously untested care concepts are introduced in the care of frail elderly persons. It also highlights the importance of complementing quantitative RCTs with qualitative assessments in a mixed methods approach, in order to capture important dimensions of healthcare quality and outcomes, analyzing complex contexts such as frail elderly care. This study confirms the strong "natural" variation in healthcare utilization among these individuals over time. The MGT intervention was clearly appreciated and highly valued but did not have the desired effects in terms of decreased hospital-based healthcare consumption, compared to the control group. As a consequence, the MGT initiative was not found to be financially self-sustainable but was highly appreciated by the patients who took advantage of it. Further long-term follow-up is needed, since it is uncertain, from this one-year follow-up, whether the MGT initiative is welfare enhancing from a societal perspective.

\section{Abbreviations}

ADL, Activities of Daily Living; CGA, Comprehensive Geriatric Assessment; CHF, Chronic Heart Failure; DiD, Difference in Difference; EMR, Emergency Room Visits; HRQoL, Health-related quality of life; MGT, Mobile Geriatric Team; MMSE, Mini Mental State Examination; RCT, Randomized Controlled Study; WMW, WilcoxonMann-Whitney.

\section{Acknowledgments}

Financial support for this study was provided by Futurum - the Academy for Healthcare, Region County council Jönköping, Sweden. We are especially grateful to RN Monica Gladh, RN Patricia Herkel, Physician Daniel Gustafsson and their coworkers in the MGT team, RN Gunnar Albinsson and last but not least, the study participants.

\section{Disclosure}

The authors report no conflicts of interest in this work.

\section{References}

1. Bloom DE, Chatterji S, Kowal P, et al. Macroeconomic implications of population ageing and selected policy responses. Lancet. 2015;385 (9968):649-657. doi:10.1016/S0140-6736(14)61464-1

2. Folkhälsomyndigheten. Folkhälsan I Sverige 2016. Arlig Rapportering [Public Health in Sweden 2016. Yearly Report]. Stockholm: Public Health Agency of Sweden; 2016. 
3. Persson G, Boström G, Allebeck P, et al. Chapter 5. Elderly people's health-65 and after. Health in Sweden: the National Public Health Report 2001. Scand J Public Health. 2001;58:117-131.

4. Dent E, Kowal P, Hoogendijk EO. Frailty measurement in research and clinical practice: a review. Eur J Intern Med. 2016;31 (SupplementC):3-10. doi:10.1016/j.ejim.2016.03.007

5. Yazdanyar A, Newman AB. The burden of cardiovascular disease in the elderly: morbidity, mortality, and costs. Clin Geriatr Med. 2009;25(4):563-vii. doi:10.1016/j.cger.2009.07.007

6. Gobbens RJ, Luijkx KG, Wijnen-Sponselee MT, Schols JM. Toward a conceptual definition of frail community dwelling older people. Nurs Outlook. 2010;58(2):76-86. doi:10.1016/j.outlook.2009.09.005

7. Swedish Association of Local Authorities and Regions. Slutrapport 2015 Sammanhållen Vård Och Omsorg Om De Mest Sjuka Äldre [Final Report 2015. Coordinated Health and Social Care for the Most Frail Older Persons.]. Stockholm: Swedish Association of Local Authorities and Regions; 2015.

8. Mazya AL, Garvin P, Ekdahl AW. Outpatient comprehensive geriatric assessment: effects on frailty and mortality in old people with multimorbidity and high health care utilization. Aging Clin Exp Res. 2019;31:519. doi:10.1007/s40520-018-1004-z

9. Pereira AA, Borim FSA, Neri AL. Risk of death in elderly persons based on the frailty phenotype and the frailty index: a review study. Revista Brasileira De Geriatria E Gerontologia. 2017;20:273-285. doi:10.1590/1981-22562017020.160102

10. Buckinx F, Rolland Y, Reginster J-Y, Ricour C, Petermans J, Bruyère O. Burden of frailty in the elderly population: perspectives for a public health challenge. Archiv Public Health. 2015;73(1):19. doi:10.1186/s13690-015-0068-x

11. Johansson L, Long H, Parker MG. Informal caregiving for elders in Sweden: an analysis of current policy developments. J Aging Soc Policy. 2011;23(4):335-353. doi:10.1080/08959420.2011.605630

12. Cardona-Morrell M, Kim JCH, Brabrand M, Gallego-Luxan B, Hillman K. What is inappropriate hospital use for elderly people near the end of life? A systematic review. Eur J Intern Med. 2017;42(SupplementC):39-50. doi:10.1016/j.ejim.2017.04.014

13. Tistad M, Ytterberg C, Sjöstrand C, Widén Holmqvist L, von Koch L. Shorter length of stay in the stroke unit: comparison between the 1990s and 2000s. Top Stroke Rehabil. 2012;19(2):172-181. doi: $10.1310 /$ tsr1902-172

14. Ellis G, Whitehead MA, Robinson D, Neill D, Langhorne P. Comprehensive geriatric assessment for older adults admitted to hospital: meta-analysis of randomised controlled trials. $B M J$. 2011;343:d6553-d6553. doi:10.1136/bmj.d6553

Clinical Interventions in Aging

\section{Publish your work in this journal}

Clinical Interventions in Aging is an international, peer-reviewed journal focusing on evidence-based reports on the value or lack thereof of treatments intended to prevent or delay the onset of maladaptive correlates of aging in human beings. This journal is indexed on PubMed Central, MedLine, CAS, Scopus and the Elsevier
15. Hayward KS. Facilitating interdisciplinary practice through mobile service provision to the rural older adult. Geriatr Nurs (Minneap). 2005;26(1):29-33. doi:10.1016/j.gerinurse.2004.11.011

16. Lifvergren S. Quality Improvement in Healthcare. Göteborg: Chalmers University of Technology; 2013.

17. Sahlen KG, Boman K, Brannstrom M. A cost-effectiveness study of person-centered integrated heart failure and palliative home care: based on a randomized controlled trial. Palliat Med. 2015;30:296302 .

18. Hasselaar J, Payne S, Sahlen K-G, Boman K, Brännström M. A costeffectiveness study of person-centered integrated heart failure and palliative home care: based on a randomized controlled trial. Palliat Med. 2016;30(3):296-302. doi:10.1177/0269216315618544

19. Launay CP, Annweiler C, Delanoe-Telfour L, de Decker L, Kabeshova A, Beauchet O. Mobile geriatric team advice: effect on length of hospital stay in older adults. J Am Geriatr Soc. 2014;62 (2):390-391. doi:10.1111/jgs.12677

20. Brorsson B, Hulter Åsberg K. Katz index of independence in ADL. Reliability and validity in short-term care. Scand J Rehab Med. 1984;16:125-132.

21. Folstein MF, Robins LN, Helzer JE. The mini-mental state examination. Arch Gen Psychiatry. 1983;40(7):812. doi:10.1001/archpsyc.1983. 01790060110016

22. Graneheim UH, Lundman B. Qualitative content analysis in nursing research: concepts, procedures and measures to achieve trustworthiness. Nurse Educ Today. 2004;24:105-112. doi:10.1016/j.nedt.2003.10.001

23. Creswell JW. A Concise Introduction to Mixed Methods Research. Thousand Oaks: SAGE; 2014.

24. Toofany S. Empowering older people. Nurs Older People. 2007;19 (2):12-14.

25. Ferrucci L, Guralnik JM, Studenski S, et al. Designing randomized, controlled trials aimed at preventing or delaying functional decline and disability in frail, older persons: a consensus report. $J$ Am Geriatr Soc. 2004;52(4):625-634. doi:10.1111/j.1532-5415.2004.52174.x

26. Ekdahl AW, Wirehn A-B, Alwin J, et al. Costs and effects of an ambulatory geriatric unit (the AGe-FIT Study): a randomized controlled trial. J Am Med Dir Assoc. 2015;16(6):497-503. doi:10.1016/ j.jamda.2015.01.074

27. Farquhar MC, Ewing G, Booth S. Using mixed methods to develop and evaluate complex interventions in palliative care research. Palliat Med. 2011;25(8):748-757. doi:10.1177/0269216311417919
Bibliographic databases. The manuscript management system is completely online and includes a very quick and fair peer-review system, which is all easy to use. Visit http://www.dovepress.com/ testimonials.php to read real quotes from published authors. 